\title{
NFD: Non-Technical Loss Fraud Detection in Smart Grid
}

\author{
Wenlin Han, Yang Xiao
}

\begin{abstract}
Utility companies consistently suffer from the harassing of Non-Technical Loss (NTL) frauds globally. In the traditional power grid, electricity theft is the main form of NTL frauds. In Smart Grid, smart meters thwart electricity theft in some ways but cause more problems, e.g., intrusions, hacking, and malicious manipulation. Various detectors have been proposed to detect NTL frauds, but they either rely on user behavior analysis which requires a large amount of historical data or needs a lot of extra devices which are expensive. In this paper, a detector, named NFD (NTL Fraud Detection), is proposed to detect NTL frauds with only a small amount of data and one additional device. NFD is based on Lagrange polynomial interpolation to model an adversary's behaviors, and detect a tampered meter by comparing the difference between the results. Different from existing detectors, our detector knows adversaries better than adversaries themselves. By building mathematical models of these adversaries, we can predict their behaviors which they may not be aware of by themselves. NFD makes it practical to detect NTL frauds both online and offline in Smart Grid. NFD can facilitate real-world applications before Smart Grid is fully deployed since it can serve the traditional power grid and Smart Grid simultaneously. Experimental results show the effectiveness of NFD. It can detect multiple tampered meters and multiple adversaries, as well as a single tampered meter and a single adversary. We also study how to tune the parameters used in NFD to further guide its practical usage.
\end{abstract}

Index Terms-Non-Technical Loss fraud, Smart Grid security, Smart meter, Malicious meter, Attacks.

\section{INTRODUCTION}

Globally, utility companies consistently suffer from NonTechnical Loss (NTL) frauds. As reported [1], the nontechnical loss even exceeds the technical loss in some countries and is estimated as about $1 \%$ of the worldwide electricity consumption. Beside electricity theft, the frauds where customers gain illegal benefit by tampering meters, intruding networks, etc., are also NTL frauds. This silence crime disturbs the normal billing process of power companies, causes money loss, and is a waste of people's efforts on energy conservation. Besides poverty and economy recession, the temptation of free service is another motivation to steal electricity in underdeveloped, developing, and developed countries, not only limited to the low-income population. As Forbes reported [2], Florida utilities lose millions each year, and thousands of NTL frauds have been reported by Duke Energy.

In the traditional power grid, the utility relies on physical methods, such as checking power lines periodically, to detect

Wenlin Han and Yang Xiao are with the Department of Computer Science, the University of Alabama, Tuscaloosa, AL 35487-0290 USA. E-mails: whan2@crimson.ua.edu, yangxiao@ieee.org.

Yang Xiao is the corresponding author, email: yangxiao@ieee.org.
NTL frauds. In Smart Grid, the development of Advanced Metering Infrastructure (AMI) enables smart meters with the capability of two-way communications. The utility can intensively monitor smart meters to detect NTL frauds remotely. Smart meters dwarf electricity theft using some physical methods, such as bypassing. However, the two-way communication extends adversaries' malicious behavior from the meters to the whole generation, transmission, and distribution process. As FBI reported [3], hacking smart meters is extremely easy and NTL frauds occur in various new forms.

Moreover, privacy protection is another challenge to detect NTL frauds in Smart Grid. Smart meters store energy usage information, and this information are distributed all over the communication networks, which potentially expose customer habits and behaviors [4]. In Smart Grid, the customers lose control over the information delivered to the utility unconsciously. Novel schemes to identify NTL without worrying about privacy are needed. Furthermore, even if customers are totally willing to provide their personal data, there's still five to ten years before AMI is fully deployed [5]. The utility must find efficient ways to detect NTL frauds right now.

Existing schemes to deal with NTL frauds can be categorized as physical methods, intrusion-detection based methods, user behavior analysis, statistic methods and comparisonbased methods. Physical methods [6], [7] are expensive and inefficient. Intrusion-detection based methods [8]-[13] can only analyze traffic and are not designed specially for NTL frauds. User behavior analysis [14]-[17] requires mining or training on a large amount of historical data. Existing statistic methods [18]-[21] are based on quantifying the experience of maintenance engineers to make a decision. But quantifying human experience is not an easy process. Comparison-based methods [22]-[25] obtain non-fraudulent measurements via inspectors and identify abnormal meters by comparing with these measurements. However, they are not cost effective when the number of inspectors is large.

In this paper, we propose a novel detector, named NFD (NTL Fraud Detection), to detect NTL frauds in Smart Grid. No additional software products are required to install on either side of Smart Grid. The detector does not require personal data, such as electricity consumption of each appliance in $24 \times$ 7 hours to analyze user behaviors, but a central observer meter for a group of consumers under investigation. This observer meter is responsible for recording the total amount of power supplied to a group during a certain time duration. Based on these data and billing electricity of each meter, we obtain mathematical models of tampered meters and normal meters according to their different characteristics which can identify 
meters with NTL frauds.

Not only NFD can detect a single meter tampered by a single adversary, but also, it can detect multiple meters tampered by multiple adversaries. NFD is based on Lagrange polynomial interpolation [27] to model an adversary's behavior, and detect a tampered meter by comparing the difference between the results. Different adversaries have different behaviors. By modeling the behaviors of adversaries, NFD can identify multiple meters tampered by multiple adversaries. With these models, NFD even knows adversaries better than adversaries themselves. The proposed detector can solve the NTL fraud problem in both Smart Grid and the traditional power grid.

The main contributions of this paper include:

- An accessible detector is proposed to detect NTL frauds without knowing any extra information of customers;

- Different from existing detectors, this detector knows adversaries better than themselves;

- This detector is a choice to serve both the traditional power grid and Smart Grid;

- This detector is practical, especially with the support of detailed discussion of parameter selection, together with theoretical proof and a case study.

The rest of the paper is organized as follows: Related work is introduced in Section II. The problem definition, the attack model, and the proposed NFD are presented in Section III. To demonstrate the effectiveness of NFD, experimental results are introduced in Section IV. The design and discussion of parameter selection, with the support of theoretical proofs, are presented in Section V. We conclude the paper and introduce future work in Section VI.

\section{RELATED WORK}

To investigate the severity of NTL frauds, various programs have been launched worldwide [28]-[37]. A study was conducted in 102 countries over an extended period of twenty years [28]. The report estimates the extent of NTL frauds and classifies them into four categories, including electricity theft, fraud, billing irregularities, and unpaid bills. In the last month of 2014, Northeast Group LLC published a report [35] showing that NTL frauds cause $\$ 89.3$ billion loss globally, among which $\$ 58.7$ billion loss is from the top 50 emerging market countries. The paper [31] presents a statistical report of NTL frauds in an Indian state. The study lasted for ten years, from 2000 to 2009. The results show an interesting statistic that electricity theft increased sharply in election years.

User behavior analysis is typically employed to detect NTL frauds [38]-[44]. The paper [39] mainly introduces the methods of feature extraction. It is a data mining method, which takes advantage of a large amount of historical data to study the behaviors of customers. These customers are classified into several types, and they have different types of user profiles. The profiles include domestic profile, industrial profile, and commercial profile. Some other factors are also taken into account, such as weather, location, etc. In the paper [38], a detection tool, named RMADS, is proposed to detect NTL frauds. Different from most existing schemes focusing on residential customers, the targets of RMADS are those large power consumers, such as factories. The basic idea of RMADS is to analyze user behavior. The main steps include: 1) generate users' load profiles on a monthly basis; 2) extract features; 3) classify the load profiles based on their features; and 4) deal with each class with a specially designed strategy. User behavior analysis relies on a large amount of historical data so that it is not functional when the data we have is limited. User behavior analysis has the potential risk of exposing users' privacy [45]-[49], such as financial situation and personal habits.

To achieve automatic identification, Artificial Intelligence (AI) is always a good choice [14]-[17], [50], [52]-[58]. The authors in [53] introduce a computational technique named Extreme Learning Machine, and an improved version - online sequential-ELM, to detect NTL frauds. It assigns random input weights and bias to kernel parameters and calculates to find a least linear solution for the linear system. It simplifies some previous research on Learning Machine, such as in the paper [54]. The online version is used to handle online applications. It is based on an NTL analysis framework to obtain and store customer load patterns during weekdays, weekends, and public holidays. Statistical methods are based on quantifying the experience of maintenance engineers to make a decision. But quantifying human experience is not an easy process.

Support Vector Machine (SVM) is a machine learning method to detect NTL frauds [14]-[16], [50], [51]. The authors in [14] first propose using SVM to detect NTL frauds. The main steps include data preprocessing, feature extraction, and classification, and this is very similar to user behavior analysis [38]. SVM is also based on user behavior analysis to detect NTL frauds, but the feature extraction and classification processes are automatic and more accurate. The authors in [15] propose an improvement to increase the convergence of SVM by combining a generic algorithm. However, it has to know abnormal behaviors that have a tight bond with NTL frauds in advance. The paper [50] proposes an improvement on the papers [14], [15]. It employs a fuzzy inference system to analyze further the customers that are classified to be abnormal. This process eliminates unusual but normal customers from the abnormal list. The methods based on AI, including SVM, require a large amount of data to train the systems and to extract features. Moreover, the training and feature extracting processes are often slow. Moreover, their classification results often have high false positive rates, since they are not good at handling variants, such as weather change, new home appliance, etc.

Alternatively, some research focus on statistical methods [18]-[21], [59]. The authors in [18] propose a statistical method to analyze the relationship between two factors: the load and the loss. It is based on the assumption that we know the total amount of losses in a distribution system in advance. Customers are classified into different categories including residential, rural, industrial, transformer, etc. By sampling from different categories, a statistical model is built to describe the relationship between the two factors: load and loss, for different bands of the coefficient $k$, which represent different regions. Based on this model, those customers with abnormal 
loss factors can be detected.

Other research related to observer meters or mathematical approaches include [22]-[26], [60]-[64]. The papers [23], [25] construct a binary tree that uses the meters as the lead nodes. The inspection process goes from the root to the leaves. It only needs one inspector (observer). However, it is not good at detecting multiple tampered meters. The paper [24] employs binary coding and permutation to detect NTL frauds. For a group of meters, it uses several inspectors (observers) to inspect each subgroup of the meters. The inspectors and meters are coded with 0 and 1 . By permutating the codes of abnormal inspectors, it can detect abnormal meters. However, it is not cost effective, since the number of inspectors it needs is half of the meters. The paper [60] introduces how to set up a remote check meter and related software for data collecting and mining. The research in [61] employs an observer meter to register total electricity supplied to several meters under observation and obtain the mathematical relationship of each meter's electricity consumption and electricity billing. It can work when the relationship is linear, and only one meter is tampered.

There are also some related work in smart grid and security as follows: communications and networking in smart grid [65]-[67], security and privacy surveys [13], [48], [75], [76], attacks [68]-[74], non-repudiation and accountability [22], [77]-[79], Vehicle-to-Grid (V2G) security [80], etc.

\section{NTL FRAUD DETECTION}

\section{A. Problem Definition and Attack Model}

As shown in Fig. 1, there is a community with $N$ households, and each household has a smart meter installed. The utility notices that the total amount of electricity registered in the billing system is always much smaller than the amount supplied to this community. The utility estimates the technical loss and deducts a part of the total loss, but the loss is still large. The utility inspects the power lines and transformers in this community for bypassing and other physical tamperings. However, no suspicious behaviors are identified. Now, NTL frauds are highly suspected, and the smart meters become new suspects.

One or more than one meters are either tampered by the customers inside the households or remotely controlled by outside adversaries. The utility needs to identify and locate these tampered meters. Or, the networks, such as AMI networks or other communication networks, are intruded by attackers. Thus, the billing data sent to the utility are falsified or altered. In this case, the utility is still interested in identifying these meters although they are not tampered. The reason is that the attackers have to obtain the keys of these meters to falsify data, and the utility has to identify these meters to replace their keys.

Thus, either locating tampered meters or detecting key exposed, the purpose is to identify those meters with issues. To make it concisely, we call these meters as tampered meters, mixing the concepts between being tampered and being intruded.

The adversaries could falsify billing data to make the amount of the billing electricity larger than the amount of the consumed. It also disturbs the normal billing process. It harms the benefit of customers since the customers have to pay more. However, it is not an NTL fraud, and thus is out of the scope of this paper. For the customers, they will never tamper meters to make the amount of the billing electricity larger than that it should be.

\section{Figure 1 is here}

\section{B. NTL Fraud Detection}

NFD needs an additional device installed to record the total electricity supplied to $n$ meters under its observation, which we call it an observer meter. In fact, at the most of the cases, such an observer meter already exist, called a head meter, e.g., in an apartment building. After further investigation, $n$ meters are highly suspected by the utility. Thus, we install a central observer meter in the same secondary distribution network with these meters, shown in Fig. 1. This observer is responsible for recording the total amount of electricity supplied to the group of $n(n \leq N)$ meters. This observer is installed outdoor and with a distance to the households since we are not allowed to observe the customers closely due to the privacy concern. Moreover, the data collected are only the usage data that the customers should provide for billing.

To introduce NFD, we first introduce some notations. Let's denote the $j$-th measured time duration as $T_{j}$. During $T_{j}$, the total amount of electricity recorded by the observer is denoted as $E_{j}$. During $T_{j}$, the amount of electricity consumed by a household $i$ is denoted as $E_{i, j}$. However, the billing amount may be a different value from $E_{i, j}$. Let's denote the billing amount as $x_{i, j}$, which is recorded by Meter $i$ during $T_{j}$. Here, we have $1 \leq i \leq n$ and $1 \leq j \leq m$. For convenience and diversity, we use three terms changeable, and they are "electricity measured", "electricity registered" and "electricity reported".

For a normal meter, the amount of electricity consumed by the household and the amount registered by this meter should be the same, which means $E_{i, j} / x_{i, j}=1$. However, there exist measurement errors. If considering measurement accuracy, $\left|E_{i, j} / x_{i, j}-1\right|$ should be very small. Let's define an accuracy ratio $\alpha_{i, j}$, which is denoted as:

$$
\alpha_{i, j}=E_{i, j} / x_{i, j}
$$

to represent the error of Meter $i$ in the time duration $j$.

We define a value range for $\alpha_{i, j}$ as $\left[\alpha_{\min }, \alpha_{\max }\right]$. Thus, we can define a meter as normal when $\alpha_{i, j} \in\left[\alpha_{\min }, \alpha_{\max }\right]$, where $(1 \leq i \leq n)$ and $1 \leq j \leq m$. A typical value of $\alpha_{m i n}$ is 0.98 and a typical value of $\alpha_{\max }$ is 1.02. Moreover, we can also believe that a meter is tampered when $\alpha_{i, j}>\alpha_{\max }$. Each meter may have different accuracy, but the difference is slight. We assume that all the meters have the same accuracy in this paper for simplicity.

$\left(x_{i, j}, i=1,2, \cdots, n\right)$ are a series of the values of the billing electricity, and their values are available. However, we do not know the values of $\left(E_{i, j}, i=1,2, \cdots, n\right)$. Thus, we cannot identify the tampered meters only by the comparison between $x_{i, j}$ and $E_{i, j}$. We notice that there is a point at the Coordinate for each value pair $\left(x_{i, j}, E_{i, j}\right)$, shown in Fig. 2. For Meter $i$, 
there are a group of value pairs related to it, and we can use the following function to represent these points:

$$
y_{i}=f_{i}(x) \text {, where } f_{i}\left(x_{i, j}\right)=E_{i, j}, j=1,2, \cdots, m \text {. }
$$

where that $f_{i}(x)$ stands for the behavior function of meter $i$ and is what we need to figure out.

Under the above assumptions, we have the following corollary, where the proof is easy, and thus is omitted.

Corollary 1. For Meter $i$, it is a tampered meter, if and only if its curve, $f_{i}(x)$, is above the line of $f(x)=\alpha_{\max } x$.

Corollary 1 can be illustrated in Fig. 2. The curve of $f_{5}(x)$ is between the lines of $f_{1}(x)=\alpha_{\max } x$ and $f_{6}(x)=\alpha_{\min } x$, and it is regarded as a normal meter. When the curves, such as the curves of $f_{2}(x), f_{3}(x)$ and $f_{4}(x)$, are above the line of $f_{1}(x)=\alpha_{\max } x$, their corresponding meters are regarded as tampered. Each point on the Coordinate of Fig. 2 is the value pair, (the measured electricity, the consumed electricity), of each meter. The functions of these curves could be

- $f(x)=a x+b$, where $a$ and $b$ are two constants, $a>$ $\alpha_{\max }, b \geq 0$

- $f(x)=x^{u}$, where $u$ is a constant;

- $f(x)=a^{x}$, where $a$ is a constant and $a>0, a \neq 1$;

- $f(x)=a \sin (b x+c)$, where $a, b$, and $c$ are three constants, and $a>0$;

- $f(x)=a \arcsin (b x+c)$, where $a, b$, and $c$ are three constants, and $a>0$;

- Other functions $f(x)>\alpha_{\max } x$.

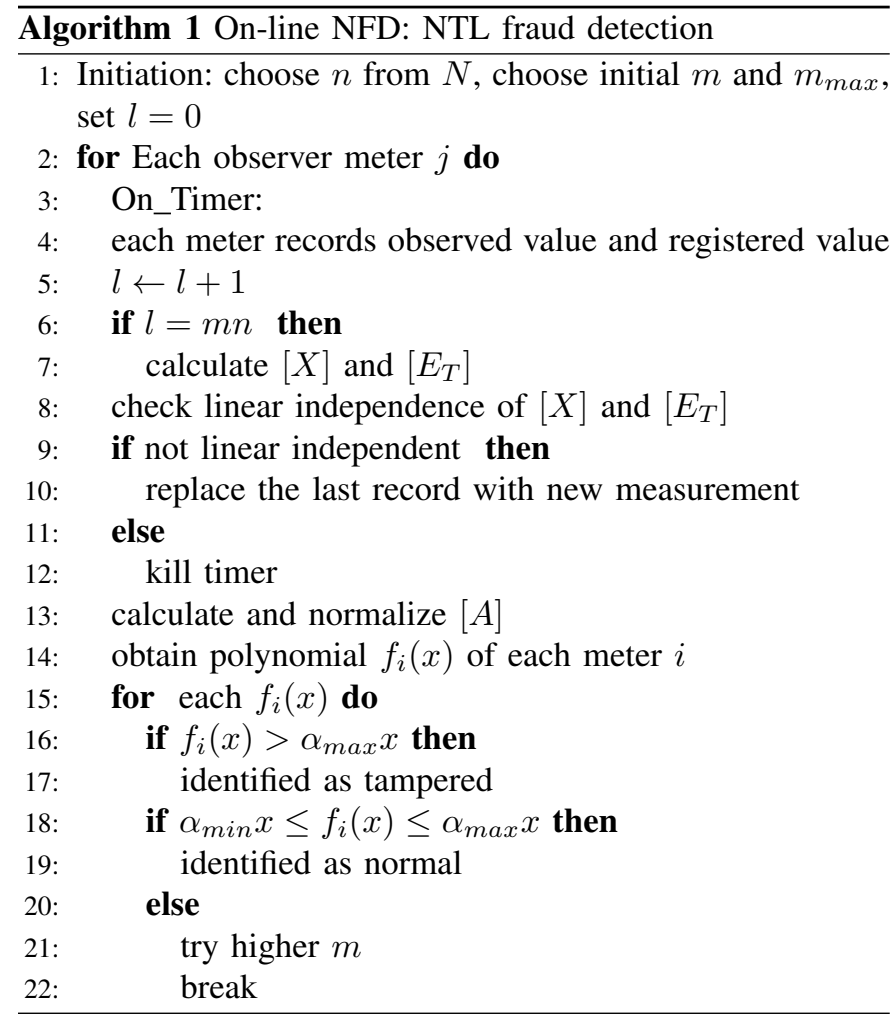

Based on Lagrange polynomials, if we have $m+1$ samples, $\left(x_{i, j}, E_{i, j}\right), 0 \leq j \leq m$, we can find an approximation function to represent them, and this function can be written as follows:

$$
f_{i}(x)=\sum_{k=m}^{0} a_{k, i} x^{k},
$$

where $a_{k, i}$ is the coefficients and $f_{i}\left(x_{i, j}\right)=E_{i, j}$.

If we know the values of the $m+1$ samples, we can obtain the coefficients $\left(a_{k, i}\right)$ by direct calculation, and thus, get the function of Meter $i$ using Eq. (3). But, till now we only get to know the values of $\left(x_{i, j}, j=1,2, \cdots, m\right)$ and we do not know the values of $\left(E_{i, j}, j=0,1, \cdots, m\right)$.

Since the distance between the observer and the meters under observation is not long, the technical loss is small enough to ignore. Under this assumption, to obtain the coefficients $\left(a_{k, i}\right), E_{j}$ can be written as:

$$
E_{j}=\sum_{i=1}^{n} E_{i, j} .
$$

Moreover, $E_{j}$, the summation of $E_{i, j}$ is available. Then, we have the following summation: $E_{j}=\sum_{i=1}^{n} E_{i, j}=$ $\sum_{i=1}^{n} f_{i}\left(x_{i, j}\right)=\sum_{i=1}^{n} \sum_{k=m}^{0} a_{k, i} x_{i, j}^{k}$.

We notice that when the amount of the billing electricity is zero, the amount of consumed electricity must be zero as well. Thus, in Eq. 3, $a_{0, i}$ should be zero. We adjust the range of $k$ to $[1, m]$. Furthermore, we re-write Eq. 4 as follows:

$$
E_{j}=\sum_{i=1}^{n} \sum_{k=m}^{1} a_{k, i} x_{i, j}^{k} .
$$

Now, the values of $x_{i, j}$ are available in Eq. (5), as well as the values of $E_{j}$. The values of all coefficients $a_{k, i}$ are unknown. Since there are $n$ meters and $m$ measurements, the total number of $a_{k, i}$ is $n \times m$.

Let's denote the array of the values of $E_{j}$ as $\mathbf{E}_{\mathbf{T}}$. Let's denote the array of all coefficients as $\mathbf{A}$. Moreover, we use $\mathbf{X}$ to represent the matrix of the measurements. Then, we have:

$$
\begin{aligned}
& \mathbf{E}_{\mathbf{T}}=\left(E_{1}, E_{2}, \cdots, E_{m}\right)^{T}, \\
& \mathbf{A}=\left(a_{m, 1}, \ldots, a_{k, 1}, \ldots, a_{1,1}, a_{0,1}, \ldots,\right. \\
& a_{m, i}, \ldots, a_{k, i}, \ldots, a_{1, i}, a_{0, i}, \ldots, \\
& \left.a_{m, n}, \ldots, a_{k, n}, \ldots, a_{1, n}, a_{0, n}\right), \\
& \mathbf{X}=\left(\begin{array}{cccccc}
x_{1,1}^{m} & \ldots & x_{1,1}^{k} & \ldots & x_{n, 1} & 1 \\
x_{1,2}^{m} & \ldots & x_{1,2}^{k} & \ldots & x_{n, 2} & 1 \\
\vdots & \vdots & \vdots & \vdots & \vdots & \vdots \\
x_{1, j}^{m} & \ldots & x_{1, j}^{k} & \ldots & x_{n, j} & 1 \\
\vdots & \vdots & \vdots & \vdots & \vdots & \vdots \\
x_{1, n m}^{m} & \ldots & x_{1, n m}^{k} & \ldots & x_{n, n m} & 1
\end{array}\right), \\
& \mathbf{E}_{\mathbf{T}}=\mathbf{X} \times \mathbf{A} \\
& \mathbf{A}=\mathbf{X}^{-1} \times \mathbf{E}_{\mathbf{T}} .
\end{aligned}
$$

The values of $\mathbf{E}_{\mathbf{T}}$ and $\mathbf{X}$ are available from the data of each measurement. Moreover, we can obtain the values of $\mathbf{X}^{-1}$ by matrix inverse of $\mathbf{X}$. Now, from Eq. (10), we get all of the 
coefficients. One constraint of the $n \times m$ equations is that they should be linearly independent. We can get all the polynomials by putting the coefficients into Eq. (3). For each meter, there exists a polynomial to represent it. By analyzing the relationship between a polynomial and $f(x)=\alpha_{\max } x$, we can identify the tampered meters. If the curve of a polynomial is above the line of $f(x)=\alpha_{\max } x$ at the Coordinate, the related meter is identified as tampered. If the curve of a polynomial is between the lines of $f(x)=\alpha_{\max } x$ and $f(x)=\alpha_{\min } x$, the related meter is identified as normal. If there is any curve of a polynomial is under the line of $f(x)=\alpha_{\min } x$, the detection process should report an error.

In this paper, we aim to detect meters which are tampered on purpose to gain illegal benefits. Thus, for each meter, its billing electricity is less than or equals to the amount of electricity consumed. Colluding attacks occur when adversaries compromise a meter so that the customer has to pay more than (s)he consumed. This problem is not what we want to solve in this paper, but it is our current research.

The proposed NFD algorithm can be used both on-line and off-line. For the off-line detection, we need to identify NTL frauds out of a certain given dataset. Since the number $(n)$ of meters and the times of measurements are already known in the dataset, what we need is to choose an appropriate order $m$, where $m \leq$ the times of measurements. For the on-line detection, we need to gather data from each measurement and identify frauds real time. The on-line detection algorithm can be seen in Algorithm 1. It will choose an initial order $m$ and then keep gathering data until it gets enough data to build an independent matrix. If the initial $m$ is not satisfying, it will try a higher $m$ and repeat the whole process until it reaches either of the following two conditions:

1) Condition 1: every meter is identified as either tampered or normal;

2) Condition 2: $m \geq m_{\max }$ and $m_{\max }$ is the maximum value of the desired order $m$.

The main steps of the detection process include preparation, calculation, normalization, and comparison. In the first step, parameters are chosen to initialize the process. In the second step, coefficients are obtained according to Alg. 1. In the third step, the results are normalized. In the final step, we compare the polynomials with $f(x)=\alpha_{\max } x$ to identify tampered meters.

We discuss parameter selection in details in Section V.

\section{EXPERIMENTS}

We conducted various experiments to test NFD, and some of the experiments are introduced in this section. We use $k W h$ as the unit for electricity values listed in the tables. The data used in our experiments are simulated. According to a report [81], the average household electricity usage around the world varies from $570 \mathrm{kWh}$ to $11879 \mathrm{kWh}$ in 2010 . The experimental data are randomly generated within this range. We manually altered some meters' usage data letting the billing amount less than the original (consumed) amount.

\section{A. Experiment 1: No tampered meters}

In this experiment, the dataset contains the data of 10 meter and an observer. We list the data of 20 measurements, including the amount of the billing electricity and the amount of the recorded value of the observer, shown in Table I.

TABLE I

THE AMOUNT OF THE BILLING ELECTRICITY (KWH) OF 10 METERS AND AN OBSERVER METER IN EXPERIMENT 1

\begin{tabular}{|c|c|c|c|c|c|c|c|c|c|c|}
\hline Meter 1 & 2 & 3 & 4 & 5 & 6 & 7 & 8 & 9 & 10 & Observer \\
\hline 4560 & 3300 & 6780 & 1900 & 2870 & 3200 & 4510 & 2800 & 4320 & 5100 & 39340 \\
\hline 5120 & 2560 & 4560 & 2440 & 1900 & 4500 & 5700 & 2120 & 5700 & 6700 & 41300 \\
\hline 3230 & 3500 & 6780 & 3900 & 1670 & 5100 & 4340 & 2000 & 5200 & 6400 & 42120 \\
\hline 6780 & 2900 & 5990 & 1890 & 2560 & 6700 & 5800 & 5300 & 6880 & 6890 & 51690 \\
\hline 6430 & 3000 & 5300 & 2120 & 4980 & 7450 & 6400 & 3050 & 4990 & 4800 & 48520 \\
\hline 1670 & 4300 & 5440 & 2800 & 5880 & 4560 & 6340 & 3560 & 4670 & 5120 & 44340 \\
\hline 2430 & 2700 & 6330 & 3000 & 2200 & 1400 & 5300 & 3650 & 4770 & 5700 & 37480 \\
\hline 7890 & 2300 & 4600 & 1900 & 2560 & 5700 & 4670 & 3670 & 2120 & 1440 & 36850 \\
\hline 8700 & 3400 & 6120 & 2650 & 2780 & 6120 & 6400 & 3100 & 5670 & 5870 & 50810 \\
\hline 4400 & 1400 & 3670 & 2990 & 2330 & 1670 & 4600 & 3540 & 5600 & 4660 & 34860 \\
\hline 5200 & 2800 & 4780 & 2110 & 5430 & 3120 & 5600 & 3000 & 5900 & 4670 & 42610 \\
\hline 3560 & 2900 & 5340 & 2440 & 5280 & 4230 & 5670 & 2890 & 4900 & 4220 & 41430 \\
\hline 2450 & 4500 & 5200 & 2660 & 3760 & 5230 & 5800 & 2400 & 2220 & 2780 & 37000 \\
\hline 4560 & 3890 & 5780 & 2770 & 4670 & 5400 & 6200 & 2670 & 3450 & 3990 & 43280 \\
\hline 3670 & 2700 & 6400 & 3120 & 4980 & 6400 & 6900 & 2670 & 3560 & 6450 & 46850 \\
\hline 3560 & 4100 & 5990 & 3450 & 4330 & 4780 & 6770 & 1990 & 4110 & 5980 & 45060 \\
\hline 2340 & 3330 & 6900 & 3200 & 3780 & 1560 & 5440 & 2500 & 4560 & 5840 & 39450 \\
\hline 5320 & 3990 & 5550 & 3320 & 4550 & 9530 & 6780 & 5700 & 4880 & 6330 & 55950 \\
\hline 3240 & 3980 & 5090 & 1990 & 2670 & 4500 & 7000 & 3890 & 4670 & 5300 & 42330 \\
\hline 4670 & 2900 & 4200 & 2550 & 4670 & 8670 & 7100 & 3600 & 5450 & 8120 & 51930 \\
\hline
\end{tabular}

In this experiment, we use the Lagrange polynomial with order 2 . Since the dataset only contains the data of 20 measurements, we cannot use an order higher than 2. Moreover, the polynomial is as follows:

$$
f_{i}(x)=a_{2, i} x^{2}+a_{1, i} x .
$$

Based on Eq. (10), we can get the values of $\mathbf{A}$. After calculation and normalization, we get

$$
\mathbf{A}=(0,1,0,1,0,1,0,1,0,1,0,1,0,1,0,1,0,1,0,1) .
$$

The obtained polynomials of all meters are shown in the second column of Table II. We compare the polynomials with $f(x)=\alpha_{\max } x$ and find that none of the meters are tampered. In other words, there are all normal meters.

\section{B. Experiment 2: Single adversary and single tampered meter}

In this experiment, the dataset contains the data of 10 meters and an observer. Table III shows the values of the amount of the billing electricity and the amount of the supplied electricity recorded by the observer in 30 measurements.

In this experiment, we employ the polynomial with an order of 3 , which is as follows:

$$
f_{i}(x)=a_{3, i} x^{3}+a_{2, i} x^{2}+a_{1, i} x .
$$

Using Eq. (10), the values of $\mathbf{A}$ can be obtained. The results after normalization are as follows:

$$
\begin{array}{r}
\mathbf{A}=(0,0,1,0,0,1,0,0,1,0,0,1,0,0,1, \\
0,0,1,0.1,0.5,1,0,0,1,0,0,1,0,0,1) .
\end{array}
$$

The polynomials of all the 10 meters in this experiments are shown in the third column of Table II. The curves of meters are shown in Fig. 3. After comparing between these polynomials and $f(x)=\alpha_{\max } x$, we notice that the curve of the $7^{\text {th }}$ meter is above the line of $f(x)=\alpha_{\max } x$. Thus, the $7^{\text {th }}$ meter is a tampered meter.

Figure 3 is here 
TABLE II

THE RESUlTS OF THE EXPERIMENTS: THE POLYNOMIALS OF ALL METERS. THERE ARE 10 METERS IN EXPERIMENTS 1 AND 2, RESPECTIVELY, AND 4 METERS IN EXPERIMENT 3.

\begin{tabular}{|c|c|c|c|c|}
\hline No. & Experiment 1 & Experiment 2 & Experiment 3 & Lowering order \\
\hline 1 & $f_{1}(x)=x$ & $f_{1}(x)=x$ & $f_{1}(x)=0.1 x^{2}+x$ & $f_{1}(x)=-0.265 x^{2}+1.308 x$ \\
\hline 2 & $f_{2}(x)=x$ & $f_{2}(x)=x$ & $f_{2}(x)=0.5 x^{2}+0.3 x$ & $f_{2}(x)=1.123 x^{2}-6.308 x$ \\
\hline 3 & $f_{3}(x)=x$ & $f_{3}(x)=x$ & $f_{3}(x)=0.1 x^{2}+0.9 x$ & $f_{3}(x)=0.006 x^{2}+0.265 x$ \\
\hline 4 & $f_{4}(x)=x$ & $f_{4}(x)=x$ & $f_{4}(x)=0.3 x^{2}+x$ & $f_{4}(x)=0.458 x^{2}-1.681 x$ \\
\hline 5 & $f_{5}(x)=x$ & $f_{5}(x)=x$ & N/A & $f_{5}(x)=-0.004 x^{2}+0.845 x$ \\
\hline 6 & $f_{6}(x)=x$ & $f_{6}(x)=0.1 x^{3}+0.5 x^{2}+x$ & N/A & $f_{6}(x)=-0.338 x^{2}+6.128 x$ \\
\hline 7 & $f_{7}(x)=x$ & $f_{7}(x)=x$ & N/A & $f_{7}(x)=1.8 x^{2}-3.32 x$ \\
\hline 8 & $f_{8}(x)=x$ & $f_{8}(x)=x$ & N/A & $f_{8}(x)=-0.42 x^{2}+3.32 x$ \\
\hline 9 & $f_{9}(x)=x$ & $f_{9}(x)=x$ & N/A & $f_{9}(x)=-0.21 x^{2}-0.41 x$ \\
\hline 10 & $f_{10}(x)=x$ & $f_{10}(x)=x$ & N/A & $f_{10}(x)=0.11 x^{2}+0.364 x$ \\
\hline
\end{tabular}

TABLE III

THE AMOUNT OF THE BILLING ELECTRICITY (KWH) OF 10 METERS AND AN OBSERVER METER IN EXPERIMENT 2

\begin{tabular}{|c|c|c|c|c|c|c|c|c|c|c|}
\hline Meter 1 & 2 & 3 & 4 & 5 & 6 & 7 & 8 & 9 & 10 & Observer \\
\hline 2110 & 3300 & 4710 & 1500 & 3000 & 8230 & 4500 & 2800 & 4600 & 5300 & 59287.5 \\
\hline 1950 & 2600 & 5330 & 2330 & 1530 & 8550 & 5600 & 4494 & 5100 & 5600 & 73951.6 \\
\hline 1800 & 3500 & 6600 & 2560 & 1990 & 9000 & 4800 & 2000 & 5200 & 6100 & 66129.2 \\
\hline 2220 & 2900 & 6780 & 1890 & 2560 & 9980 & 5120 & 5000 & 6780 & 6990 & 76749 \\
\hline 2300 & 3000 & 4990 & 2120 & 4560 & 8770 & 6000 & 3050 & 4900 & 4670 & 83960 \\
\hline 1570 & 3700 & 6100 & 2890 & 5660 & 8990 & 6340 & 3500 & 4880 & 5120 & 94331.8 \\
\hline 2500 & 2700 & 6230 & 3000 & 2200 & 7990 & 5300 & 3600 & 4780 & 5330 & 72562.7 \\
\hline 2300 & 3200 & 4780 & 1900 & 2560 & 8230 & 4990 & 3670 & 2100 & 5900 & 64505.2 \\
\hline 2880 & 3400 & 5330 & 2440 & 2890 & 8450 & 4890 & 3090 & 5800 & 5810 & 68629.1 \\
\hline 1930 & 3100 & 3550 & 2670 & 2330 & 8650 & 4600 & 3550 & 5700 & 4890 & 61283.6 \\
\hline 2120 & 2500 & 4880 & 2110 & 5330 & 8100 & 4500 & 2990 & 5550 & 4550 & 61867.5 \\
\hline 2300 & 2900 & 4940 & 2230 & 5280 & 7450 & 5670 & 2890 & 4900 & 4200 & 77062.9 \\
\hline 2000 & 2900 & 5120 & 2660 & 3600 & 7810 & 5800 & 2700 & 2100 & 2600 & 736212 \\
\hline 1750 & 4000 & 5440 & 1990 & 4670 & 9110 & 6200 & 2560 & 3600 & 3900 & 86272.8 \\
\hline 1820 & 2700 & 5980 & 3120 & 4880 & 9300 & 6900 & 2670 & 3700 & 6230 & 103955.9 \\
\hline 2740 & 2760 & 5770 & 3450 & 4330 & 8560 & 6770 & 1900 & 4100 & 6430 & 100755.3 \\
\hline 2450 & 3330 & 4890 & 3330 & 3990 & 8440 & 5440 & 2500 & 4800 & 5810 & 75875.7 \\
\hline 2530 & 3670 & 5220 & 3320 & 4550 & 9400 & 6450 & 3060 & 4900 & 6300 & 97034.9 \\
\hline 2320 & 3980 & 5540 & 1970 & 2550 & 9910 & 6340 & 3560 & 4780 & 5900 & 92431.8 \\
\hline 2780 & 2990 & 5900 & 2550 & 3890 & 8760 & 6100 & 3880 & 5600 & 5660 & 89413.1 \\
\hline 2300 & 3450 & 6780 & 1770 & 3200 & 7770 & 5990 & 3660 & 1200 & 1900 & 77452.2 \\
\hline 3450 & 4320 & 7120 & 1800 & 3560 & 7650 & 5870 & 3400 & 1340 & 1980 & 77944.7 \\
\hline 5320 & 2450 & 8120 & 2890 & 4120 & 8010 & 5770 & 3080 & 2450 & 200 & 78266.5 \\
\hline 4340 & 3090 & 7890 & 3120 & 4030 & 7990 & 5050 & 2090 & 2980 & 4500 & 70710 \\
\hline 2630 & 4670 & 6120 & 3450 & 2120 & 7120 & 6120 & 4090 & 2100 & 5230 & 85299.3 \\
\hline 3120 & 5120 & 6660 & 3200 & 2670 & 6890 & 4890 & 3900 & 3210 & 6120 & 69429.1 \\
\hline 3090 & 3100 & 6450 & 4300 & 3400 & 6770 & 4990 & 3890 & 4500 & 5900 & 71265.2 \\
\hline 1980 & 3980 & 5700 & 3780 & 3080 & 6080 & 5340 & 3100 & 4090 & 4980 & 71595.1 \\
\hline 2890 & 4560 & 7230 & 3880 & 3030 & 6130 & 5220 & 2030 & 1900 & 4870 & 69587.9 \\
\hline 2770 & 3900 & 7450 & 3200 & 3980 & 7450 & 5090 & 2450 & 2890 & 800 & 66121.3 \\
\hline
\end{tabular}

\section{Experiment 3: Multiple adversaries and multiple tampered meters}

In this experiment, the dataset contains the data of 4 meters and an observer. The amount of the billing electricity and the amount of the supplied electricity in 8 measurements are listed in Table IV. We employ the polynomial with an order of 2, which is the same as in Exp. 1.

TABLE IV

THE AMOUNT OF THE BILLING ELECTRICITY (KWH) OF 4 METERS AND AN OBSERVER METER IN EXPERIMENT 3

\begin{tabular}{|c|c|c|c|c|}
\hline Meter 1 & Meter 2 & Meter 3 & Meter 4 & Observer Meter \\
\hline 2100 & 4000 & 3000 & 6700 & 35508 \\
\hline 1800 & 5000 & 4000 & 10300 & 63451 \\
\hline 2500 & 3600 & 8000 & 4500 & 34860 \\
\hline 1900 & 5300 & 6000 & 5600 & 41904 \\
\hline 2200 & 6300 & 7000 & 8900 & 68282 \\
\hline 2600 & 4000 & 4500 & 9400 & 54459 \\
\hline 3000 & 5000 & 3400 & 8700 & 53523 \\
\hline 1700 & 3000 & 3700 & 8600 & 42876 \\
\hline
\end{tabular}

Now we can obtain A, according to Eq. (10). After calculation and normalization, we get

$$
\mathbf{A}=(0.1,1,0.5,0.3,0.1,0.9,0.3,1) .
$$

The obtained polynomials of all the meters in this experiment can be seen in the fourth column of Table II. After comparing these polynomials with $f(x)=\alpha_{\max } x$, we find that all the meters are tampered meters. The curves of these meters, with a comparison to the normal meter, are shown in Fig. 3.

\section{PARAMETER SELECTION AND DISCUSSION}

The selection of the order $m$ is decided by accuracy requirement. If a higher accuracy is required, a bigger $m$ is needed, at the most of the time. Also, more measurement data are needed to obtain a polynomial with a higher order of $m$. However, a bigger $m$ not always results in a higher accuracy.

In this section, some discussions will be carried out to guide applicable usage of this scheme. Theoretical analysis and related proofs will be carried out, together with some case studies. Its main purpose is to answer the following questions:

- How to choose the order $m$ ?

- For a given order $m$, what should the error be?

- To lower the error, should we choose a higher $m$ ?

- For a given order $m$, how many measurement data do we need?

- How many meters should be in the same group (how to choose $n)$ ?

- How much detection time needed to identify a fraud?

- How many observer meters needed in a community?

\section{A. Error and selection of order $m$}

In this subsection, we'll discuss the relationship between the order $m$ and the corresponding error. Related theoretical analysis and case study will help to choose a better value of the order $m$.

Now, we'll use the same dataset in Experiment 2 to illustrate the error. In Experiment 2, we use an order 3 polynomial to fit the dataset. Here, we try to use an order 2 polynomial to fit the dataset. The result is shown in Table II.

From Table II, the last column, maybe it is hard to identify the tampered one. But it is easy to differentiate the tampered meter from those normal meters in Fig. 4. The black line is the normal meter with the polynomial of $f(x)=\alpha_{\max } x$. The lines or curves above this black one are suspected to be tampered. We can see that only the curves of Meter 6 and Meter 7 are above it. Moreover, for Meter 6, its slope 
rate is almost the same as the normal meter. Meter 7's curve is far from the normal one with a sharper slope. Meter 7 is tampered, as the result of Experiment 2. But for Meter 6 , it is a false alarm. The reason is that to get the order 2 polynomial, we only need 20 out of the total 30 measurements dataset in Experiment 2. The occurrence of error is caused by lower accuracy. Reversely, to identify the tampered meters accurately, more data or measurements are needed.

The comparison of the order 2 and order 3 curves of the same meter - Meter 7, is shown in Figs. 3(e) and (f). The range of $x$ in Fig. 3(e) is the same as in the experimental dataset. Fig. 3(f) shows the curves where $x$ is in $[0,10]$.

The error between the approximation polynomial and its original function, according to Lagrange remainder theorem [82], can be given as:

$$
R_{n}(x)=\frac{f^{n+1}(\xi)}{(n+1) !}\left(x-x_{0}\right)^{n+1}, \xi \in\left[x_{0}, x\right] .
$$

However, we cannot get to know the original form of the function only from two of its approximation polynomials. Here, we propose another method, to estimate the error of lowering order. Let's define:

$e$ is the error of lowering the order;

$x_{i}$ is the value of the measured electricity in each Meter $i$

$f_{1}\left(x_{i}\right)$ is the corresponding value of $x_{i}$ in the lowerorder polynomial $f_{1}(x)$;

$f_{2}\left(x_{i}\right)$ is the corresponding value of $x_{i}$ in the higherorder polynomial $f_{2}(x)$;

$n$ is the number of sample points obtained from each of the polynomials.

Definition 1. Assume that a meter has two polynomials, $f_{1}(x)$ and $f_{2}(x)$, which are obtained from the same dataset of measurements, where $f_{2}(x)$ is the higher-order polynomial and $f_{1}(x)$ is the lower-order polynomial. Their relative error is given by:

$$
e=\sqrt{\frac{\sum_{x_{i}=x_{1}}^{x_{n}}\left(\frac{f_{1}\left(x_{i}\right)-f_{2}\left(x_{i}\right)}{f_{1}\left(x_{i}\right)}\right)^{2}}{n}} .
$$

Conjecture 1. The error - e given in Definition 1 can describe the relative error of two polynomials with different orders obtained from the same dataset. Its accuracy is in the same order of magnitude of Lagrange remainder as given in Eq. (13).

Now consider the function $f(x)=2 \sin (x)$. Its Taylor approximation, when $x_{0}=0$, is as follows:

- Order-1: $f(x)=2 x$;

- Order-2: $f(x)=2 x$;

- Order-3: $f(x)=2 x-1 / 3 x^{3}$;

- Order-4: $f(x)=2 x-1 / 3 x^{3}$;

- Order-5: $f(x)=2 x-1 / 3 x^{3}+1 / 60 x^{5}$.

We calculate the relative error according to Eq. (14) and Eq. (13). Note that in Eq. (13), we set $x_{0}=0$, and calculate its relative error. For example, the relative error of the order 5
TABLE V

COMPARISON OF THE ACCURACY MAGNITUDE BETWEEN OUR DEFINITION AND LAGRANGE REMAINDER.

\begin{tabular}{|c|c|c|c|}
\hline Operation & Our Definition & Taylor & Accuracy Mag. \\
\hline Order $3 \rightarrow$ order 2 & $\mathrm{e}=0.29$ & $\mathrm{e}=0.13$ & $10^{-1}$ \\
\hline Order $5 \rightarrow$ order 4 & $\mathrm{e}=0.028$ & $\mathrm{e}=0.014$ & $10^{-2}$ \\
\hline
\end{tabular}

and the order 4 is $R_{5}(x)-R_{4}(x)$. From the result shown in Table V, their accuracies are in the same order of magnitude. Although we cannot prove it now, since we do not know the original form of the function, this conjecture still can guide some practical usage of the scheme. According to Eq. (14), the error in our experiment of lowering the order is $4 \%$.

Thus for a certain amount of given datasets (for off-line detection), how to determine the order of the polynomial needed to identify a fraud? Based on the results of Experiment 3, most people may believe to get the most accurate polynomial, the best is to use the whole dataset of all measurements. That is

$$
m=\lfloor(l / n)\rfloor \text {. }
$$

However, a higher order $m$ does not result in a higher accuracy sometimes. Now suppose that:

- The number of meters is $n$;

- The total time of measurements is $l$, only refer to the measurements with complete data;

- The needed order of the polynomial is $m$.

Theorem 2. Obtaining more measurements or using a larger historical dataset to obtain a polynomial with a higher order does not always improve the accuracy of the polynomial.

Proof: see Appendix A.

Theorem 2 tells us that a higher order $m$ does not always increase the detection accuracy. In Theorem 2, we present two lemmas. Lemma 5 tells us when a higher order $m$ can increase the detection accuracy, and Lemma 6 tells us when a higher order $m$ cannot increase the detection accuracy.

Under some circumstances, if the dataset is too large, we need to choose a subset of the data measured, and to get a relatively lower but good $m$. The criterion that we propose is to constrain the error - $e$, as in Eq. (14), within the accuracy class. That is

$$
e<|\alpha-1|
$$

Under some circumstances, if the dataset is too small for more accurate analysis, or we need a shorter detection time, we can ignore some coefficients, to get a better result with limited data.

Lemma 3. With limited data gathered, ignoring $a_{0}$ can improve accuracy and save detection time. That is: when $l \leq 3 n$, where $l$ is the number of measurements with complete data gathered,

$f_{i}(x)=a_{m, i} x^{m}+a_{m-1, i} x^{m-1}+\cdots+a_{k, i} x^{k}+\cdots+a_{1, i} x$.

Proof: Compare it to Eq. (3). For $n$ meters, Eq. (17) needs $n$ fewer times of measurements to get the result so that it will save detection time. Moreover, based on the same dataset, 
with Eq. (17) we can get one order higher than with Eq. (3). According to Lemma 5 (see Appendix A), with Eq. (17), we can get a higher accuracy. Note that the situation mentioned in Lemma 6 (see Appendix A), only occurs in high order approximation, rather than $\leq 5$ order.

For most on-line cases, $m$ can be chosen as 2 initially, and according to Alg. 1, $m$ can be adjusted to a higher order towards $m_{\max }$. In most off-line cases, $m_{\max }$ can be chosen as 3 to 5 , according to different sizes of datasets. Typically, an order of $m_{\max }=5$ is enough for most practical applications.

\section{B. Detection time and selection of user number $n$}

In this subsection, we will discuss fraud detection time, how to select $n$ out of the total $N$ users or meters in a community, and the number of observer meters needed.

The detection time $t$ is decided by the desired order $m$, the number of meters under observation $n$, and the time interval of measurement $T_{0}$. It's given as

$$
t=m n T_{0}
$$

In Smart Grid, if we expect to detect NTL frauds within 6 hours, where the metering interval is set to be 15 minutes typically [75], one observer meter can observe 8 meters at most, and the desired $m$ is 3 . In a community with $N$ meters, at least, $\lceil N / 8\rceil$ observer meters should be installed. Inversely, if consider from the angle of saving budget, installing one observer meter for each community is considerable. In the traditional power grid, the time interval for measurement may be much longer than in Smart Grid, because of the difficulty of gathering data. If we can get data once per day, we need at least 24 days to achieve the same effect as in Smart Gird. Now consider saving the detection time and the budget at the same time. We can set different weights or priorities for the detection time and the budget.

Let's denote $w_{1}$ as the weight of the detection time and $w_{2}$ as the weight of the number of the observer meters, respectively. Here, fewer observer meters means less budget needed. We define $o$ as the number of the observer meters installed in a community.

Theorem 4. To balance the budget and the detection time in $N F D$, the number of the observer meters o must satisfy the following equation:

$$
o=\sqrt{\frac{w_{1} m N T_{0}}{w_{2}}},
$$

and the number of meters $n$ monitored by one observer cannot exceed:

$$
n \leq \sqrt{\frac{N w_{2}}{w_{1} m T_{0}}} .
$$

Proof: Let's define $y$ as:

$$
y=w_{1} t+w_{2} o
$$

To balance the budget and the detection time in NFD, $y$ should be minimized. We notice that

$$
n=N / o \text {, }
$$

and put $n$ into Eq. (18), we get

$$
t=m N T_{0} / o \text {. }
$$

Now, put $t$ into Eq. (21), we get

$$
\begin{aligned}
y & =w_{1} t m N T_{0} / o+w_{2} o \\
& \geq 2 \sqrt{w_{1} m N T_{0} w_{2}} .
\end{aligned}
$$

When $o=\sqrt{\frac{w_{1} m N T_{0}}{w_{2}}}, y$ is the minimal, and the value is $2 \sqrt{w_{1} m N T_{0} w_{2}}$. Moreover, the related detection time $t$ is $\frac{m N T_{0} w_{2}}{w_{1}}$.

Correspondingly, according to Eq. (22), the number of meters monitored by one observer cannot exceed $\sqrt{\frac{N w_{2}}{w_{1} m T_{0}}}$.

\section{CONCLUSION}

In this paper, a novel detector NFD is proposed to detect NTL frauds in Smart Grid. NFD is based on Lagrange polynomials to generate a polynomial for each meter using a small dataset. By comparing the polynomials between the normal meters and the abnormal meters, we can identify tampered meters. Various experiments have been conducted to show the effectiveness of NFD. A detailed discussion about parameter selection, together with mathematical proofs and case study, have guided its way to real-world practical applications. As a future work, we will further study the conjecture in this paper. Moreover, for the on-line detection algorithm, we will set up a series of experiments to test its false alarm rate and efficiency in real-world applications.

Note that our paper does not like a traditional security paper such as intrusion detection system (IDS) paper where how to choose a good detection threshold is an important aspect. Instead, our paper uses a non-traditional approach to tackle a security problem. Our focus is not how to choose a better detection threshold, but presenting our method and the foundation of the method in general. How to choose a better threshold will be our future research.

\section{ACKNOWLEDGEMENT}

This work was partially supported by the National Science Foundation (NSF) under grant CNS-1059265, and by the National Natural Science Foundation of China under the grant 61374200 .

\section{APPENDIX A}

PROOF OF THEOREM 2

Proof: Suppose that for a given Meter $i$, its approximation polynomial is $Q_{i}(x)$ with the order of $m$, its approximation polynomial is $P_{i}(x)$ with the order of $m+1$, and its original function is $f_{i}(x)$. According to Eq. (13), the error of $Q_{i}(x)$ is as follows:

$$
R_{i, m}(x)=\frac{f^{(m+1)}(\xi)}{(m+1) !}\left(x-x_{0}\right)^{m+1}, \xi \in\left[x_{0}, x\right] .
$$

Lemma 5. When $\lim _{m \rightarrow \infty} R_{i, m}(x)=0$, the accuracy of $P_{i}(x)$ is always higher than $\stackrel{m \rightarrow \infty}{Q_{i}}(x)$. 
Proof: For

$$
f_{i}(x)=Q_{i}(x)+R_{i, m}(x),
$$

and $Q_{i}(x)=\sum_{j=0}^{m} \frac{f_{i}^{(j)}\left(x_{0}\right)}{j !}\left(x-x_{0}\right)^{j}, R_{i, m}(x)$ can be given as

$R_{i, m}(x)=\frac{f_{i}^{(m+1)}\left[x_{0}+\theta\left(x-x_{0}\right)\right]}{(m+1) !}\left(x-x_{0}\right)^{m+1}, 0<\theta<1$.

Putting it into Eq. (26), we get

$$
\begin{aligned}
f_{i}(x) & =\sum_{j=0}^{m} \frac{f_{i}^{(j)}\left(x_{0}\right)}{j !}\left(x-x_{0}\right)^{j}+\frac{f_{i}^{(m+1)}\left[x_{0}+\theta\left(x-x_{0}\right)\right]}{(m+1) !} . \\
\left(x-x_{0}\right)^{m+1} & .
\end{aligned}
$$

For $f_{i}^{(m+1)}(x)$, apply mean-value theory [83] in the close interval $\left[x_{0}, x_{0}+\theta\left(x-x_{0}\right)\right]$, we get

$$
\frac{f_{i}^{(m+1)}\left[x_{0}+\theta\left(x-x_{0}\right)\right]-f_{i}^{(m+1)}\left(x_{0}\right)}{\theta\left(x-x_{0}\right)}=f_{i}^{(m+2)}(\xi) .
$$

Thus,

$f_{i}^{(m+1)}\left[x_{0}+\theta\left(x-x_{0}\right)\right]=\theta\left(x-x_{0}\right) f_{i}^{(m+2)}(\xi)+f_{i}^{(m+1)}\left(x_{0}\right)$.

Put Eq. (30) into Eq. (28) to obtain:

$$
f_{i}(x)=Q_{i,(m+1)}(x)+\frac{\theta f_{i}^{(m+2)}(\xi)}{(m+1) !}\left(x-x_{0}\right)^{m+2} .
$$

From Eq. (26), we get

$$
f_{i}(x)=Q_{i,(m+1)}(x)+\frac{f_{i}^{(m+2)}\left[x_{0}+\theta_{1}\left(x-x_{0}\right)\right]}{(m+2) !}\left(x-x_{0}\right)^{m+2},
$$$$
0<\theta_{1}<1 \text {. }
$$

Comparing Eqs. (31) and (32), the following can be obtained

$$
\begin{aligned}
\frac{\theta f_{i}^{(m+2)}(\xi)}{(m+1) !}\left(x-x_{0}\right)^{m+2} & =\frac{f_{i}^{(m+2)}\left[x_{0}+\theta_{1}\left(x-x_{0}\right)\right]}{(m+2) !} . \\
\left(x-x_{0}\right)^{m+2} & .
\end{aligned}
$$

According to [84], suppose $x \rightarrow x_{0}$.

$$
\begin{aligned}
& \because f_{i}^{(m+2)}\left(x_{0}\right) \neq 0, \text { and } f_{i}^{(m+2)}(x) \text { is continuous, } \\
& \therefore \lim _{x \rightarrow x_{0}} \theta=\frac{1}{m+2} .
\end{aligned}
$$

When $x_{0}$ is in a sufficiently small neighborhood, $\theta \approx \frac{1}{m+2}$, and

$$
f_{i}(x) \approx Q_{i, m}(x)+\frac{f_{i}^{(m+1)}\left(x_{0}+\frac{x-x_{0}}{m+2}\right)}{(m+1) !}\left(x-x_{0}\right)^{m+1} .
$$

The right side of Eq. (33) is actually the approximation of $f_{i}(x)$ with $m+1$ order. That is

$$
P_{i}(x)=Q_{i, m}(x)+\frac{f_{i}^{(m+1)}\left(x_{0}+\frac{x-x_{0}}{m+2}\right)}{(m+1) !}\left(x-x_{0}\right)^{m+1},
$$

and $Q_{i, m}(x)=Q_{i}(x)$. Thus,

$$
\begin{aligned}
& f_{i}(x)-P_{i}(x)=f_{i}(x)-Q_{i}(x)-\frac{f_{i}^{(m+1)}\left(x_{0}+\frac{x-x_{0}}{m+2}\right)}{(m+1) !} . \\
& \left(x-x_{0}\right)^{m+1} \\
= & \frac{f_{i}^{(m+1)}\left(x_{0}\right)}{(m+1) !}\left(x-x_{0}\right)^{m+1}+\frac{f_{i}^{(m+2)}\left[x_{0}+\left(\theta_{1}\left(x-x_{0}\right)\right)\right]}{(m+2) !} . \\
& \left(x-x_{0}\right)^{m+2}-\frac{f_{i}^{(m+1)}\left(x_{0}+\frac{x-x_{0}}{m+2}\right)}{(m+1) !}\left(x-x_{0}\right)^{m+1} \\
= & \frac{f_{i}^{(m+2)}\left[x_{0}+\left(\theta_{1}\left(x-x_{0}\right)\right)\right]}{(m+2) !}\left(x-x_{0}\right)^{m+2}-\frac{\left(x-x_{0}\right)^{m+1}}{(m+1) !} . \\
& \int_{x_{0}}^{x_{0}+\frac{x-x_{0}}{m+2}} f^{m+2}(t) d t \\
= & \frac{\left(x-x_{0}\right)^{m+1}}{(m+1) !}\left\{\frac{f_{i}^{m+2}\left[x_{0}+\theta_{1}\left(x-x_{0}\right)\right]}{m+2}\left(x-x_{0}\right)\right. \\
& \left.-\int_{x_{0}}^{x_{0}+\frac{x-x_{0}}{m+2}} f^{m+2}(t) d t\right\}, \theta_{1} \in(0,1) .
\end{aligned}
$$

According to the mean value theorem of integrals, there exists $\eta \in\left(x_{0}, x_{0}+\frac{x-x_{0}}{m+2}\right)$, and

$$
\begin{aligned}
f_{i}(x)-P_{i}(x) & =\frac{\left(x-x_{0}\right)^{m+2}}{(m+2) !}\left\{f^{(m+2)}\left[x_{0}+\theta_{1}\left(x-x_{0}\right)\right]\right. \\
& \left.-f^{(m+2)}(\eta)\right\} .
\end{aligned}
$$

- When $f^{(m+2)}\left[x_{0}+\theta_{1}\left(x-x_{0}\right)\right]-f^{(m+2)}(\eta) \neq 0, f_{i}(x)-$ $P_{i}(x)=\mathcal{O}\left(\left(x-x_{0}\right)^{m+2}\right)$

- When $f^{(m+2)}\left[x_{0}+\theta_{1}\left(x-x_{0}\right)\right]-f^{(m+2)}(\eta)=0, f_{i}(x)-$ $P_{i}(x)=o\left(\left(x-x_{0}\right)^{m+2}\right)$.

Therefore, the accuracy of $P_{i}(x)$ is always higher than $Q_{i}(x)$.

Lemma 6. When $\lim _{m \rightarrow \infty} R_{i, m}(x)=+\infty$, the accuracy of $P_{i}(x)$ is not always higher than $Q_{i}(x)$.

Proof: We prove it with a counterexample.

Consider the following function

$$
f_{i}(x)=\frac{2}{2+33 x^{2}} .
$$

Figure 5 is here

Fig. 5 shows its approximation polynomials with order 2 , order 3 , order 5 , and order 9 , respectively. The order 5 polynomial, the red curve, is a better approximation than the order 2, the green curve, and the order 3, the magenta curve. Most of the order 9 curve, the blue one, is closer to the original function than the order 5, the red curve. However, it oscillates at the end of the interval, i.e. close to -1 and 1 . The reason is that the upper bound for the approximation error grows to infinity with $m$ [85].

$$
f_{i}(x)-Q_{i}(x)=\frac{f^{(m+1)}(\xi)}{(m+1) !} \prod_{j=1}^{m+1}\left(x-x_{j}\right), \xi \in\left[x_{0}, x\right],
$$


and for some $\xi \in(-1,1)$,

$$
\begin{array}{r}
\max _{-1 \leq x \leq 1}\left|f_{i}(x)-P_{i}(x)\right| \leq \max _{-1 \leq x \leq 1} \frac{f_{i}^{(m+1)}(x)}{(m+1) !} . \\
\max _{-1 \leq x \leq 1} \prod_{j=1}^{m+1}\left(x-x_{j}\right) .
\end{array}
$$

Thus,

$$
\lim _{m \rightarrow \infty}\left(\max _{-1 \leq x \leq 1}\left|f_{i}(x)-P_{i}(x)\right|\right)=+\infty .
$$

Because of the oscillation, a meter's polynomial of a higher order could be less accurate than its polynomial of a lower order, especially when the range of the measured electricity - $x$, is close to the oscillation range. Therefore, when $\lim _{m \rightarrow \infty} R_{i, m}(x)=+\infty$, the accuracy of $P_{i}(x)$ is not always higher than $Q_{i}(x)$.

Based on Lemmas 5 and 6 ,

1) When $\lim _{m \rightarrow \infty} R_{i, m}(x)=0$, the accuracy of $P_{i}(x)$ is always higher than $Q_{i}(x)$;

2) When $\lim _{m \rightarrow \infty} R_{i, m}(x)=+\infty$, the accuracy of $P_{i}(x)$ is not always higher than $Q_{i}(x)$.

A polynomial with a higher order $m$ does not always improve the accuracy of its approximation. Theorem 2 has then been established.

\section{REFERENCES}

[1] R. Targosz, "Electricity Theft - A Complex Problem," available at: http://www.leonardo-energy.org/electricity-theft-complexproblem, Jul. 2009.

[2] K. Silverstein, "India and U.S. Share Energy Woes: Stealing Electricity," available at: http://www.forbes.com/sites/kensilverstein/2012/08/05/indiaand-u-s-share-energy-woes-stealing-electricity/, 2012

[3] K. Tweed, "FBI Finds Smart Meter Hacking Surprisingly Easy," available at: http://www.greentechmedia.com/articles/read/fbi-findssmart-meter-hacking-surprisingly-easy/, 2012

[4] P. McDaniel, "Security and Privacy Challenges in the Smart Grid," IEEE Security \& Privacy, Vol. 7, No. 3, pp. 75-77, 2009.

[5] IEE report, "Utility-scale Smart Meter Deployments, Plans, \& Proposals," available at: http://www.edisonfoundation.net/iee/Documents/ IEE SmartMeterRollouts_0512.pdf, May 2012.

[6] P. Rengaraju, S. R. Pandian, and C. Lung, "Communication Networks and Non-Technical Energy Loss Control System for Smart Grid Networks," In Proc. of the 2014 IEEE Innovative Smart Grid Technologies - Asia (ISGT ASIA), pp. 418-423, May 2014.

[7] I. H. Cavdar, "A Solution to Remote Detection of Illegal Electricity Usage via Power Line Communications," IEEE Transactions on Power Delivery, Vol. 19, No. 4, Oct. 2004.

[8] D.J. Gaushell and H.T. Darlington, "Supervisory Control and Data Acquisition," In Proc. of the IEEE, Vol. 75, No. 3, pp. 1645-1658, 2005.

[9] R. Berthier and W. H. Sanders, "Monitoring Advanced Metering Infrastructures with Amilyzer," Cyber-security of SCADA \& industrial control systems, 2013.

[10] S. McLaughlin, B. Holbert, S. Zonouz, and R. Berthier, "AMIDS: A Multi-Sensor Energy Theft Detection Framework for Advanced Metering Infrastructures," In Proc. of the 2012 IEEE Third International Conference on Smart Grid Communications (SmartGridComm'12), pp. 354-359, Nov. 2012.

[11] Z. A. Baig, "Rapid Anomaly Detection for Smart Grid Infrastructures through Hierarchical Pattern Matching," International Journal of Security and Networks (IJSN), Vol. 7, No. 2, pp. 83-94, 2012.

[12] C. D'Orazio and K. Choo, "An adversary model to evaluate DRM protection of video contents on iOS devices," Computers \& Security, Vol. 56, pp. 94-110, Feb. 2016.

[13] J. Gao, J. Liu, B. Rajan, R. Nori, B. Fu, Y. Xiao, W. Liang, and C. L. P. Chen, "SCADA Communication and Security Issues," (Wiley Journal of) Security and Communication Networks, Vol. 7, No. 1, pp. 175-194, 2013.
[14] J. Nagi, K. Yap, S. Tiong, S. Ahmed, and M. Mohamad, "Nontechnical Loss Detection for Metered Customers in Power Utility Using Support Vector Machines," IEEE Transaction on Power Delivery, Vol. 25, No. 2, pp. 1161-1171, Dec. 2010.

[15] J. Nagi, K. Yap, S. Tiong, S. Ahmed, and A. M. Mohammad, "Detection of Abnormalities and Electricity Theft Using Genetic Support Vector Machines," In Proc. IEEE Region 10 Conference, pp. 1, Nov. 2008.

[16] Depuru S.S.S.R., L. Wang, and V. Devabhaktuni, "Support Vector Machine Based Data Classification for Detection of Electricity Theft," In Proc. IEEE/PES Power Systems Conference and Exposition (PSCE), pp. 1-8, Phoenix, USA, Mar. 2011.

[17] Z. Markoc, N. Hlupic, and D. Basch, "Detection of suspicious patterns of energy consumption using neural network trained by generated samples," In Proc. 33rd International Conference on Information Technology Interfaces (ITI), Jun. 2011.

[18] M. E. de Oliveira, D. F. A. Boson, and A. Padilha-Feltrin, "A Statistical Analysis of Loss Factor to Determine the Energy Losses," In Proc. IEEE/PES Transmission and Distribution Conference and Exposition: Latin America, pp. 1-6, Bogota, Distrito, Aug. 2008.

[19] S. Ribeiro, R. Janeiro, T. Cazes, R. Mano, and D. Maia, "Investment optimization methodology applied to investments on non-technical losses reduction actions," In Proc. IEEE Symposium on Computers and Communications (ISCC), Jul. 2012.

[20] I. Monedero, F. Biscarri, C. Len, J. I. Guerrero, J. Biscarri, and R. Milln, "Detection of Frauds and Other Non-technical Losses in a Power Utility Using Pearson Coefficient, Bayesian Networks and Decision Trees," Electrical Power and Energy Systems, Vol. 34, No. 1, pp. 90-98, Jan. 2012.

[21] C. Ramos, A. Souza, J. Papa, and A. Falco, "A New Approach for Nontechnical Losses Detection Based on Optimum-path Forest," IEEE Transactions on Power System, Vol. 26, No. 1, pp. 181-189, 2011.

[22] Z. Xiao, Y. Xiao, and D. Du, "Non-repudiation in Neighborhood Area Networks for Smart Grid," IEEE Communications Magazine, Vol. 51, No. 1, pp. 18-26, Jan. 2013.

[23] Z. Xiao, Y. Xiao, and D. Du, "Exploring Malicious Meter Inspection in Neighborhood Area Smart Grids," IEEE Transactions on Smart Grid, Vol. 4, No. 1, pp. 214-226, Mar. 2013.

[24] X. Xia, W. Liang, Y. Xiao, and M. Zheng, "BCGI: A Fast Approach to Detect Malicious Meters in Neighborhood Area Smart Grid," In Proc. of the 50th International Conference on Communications (ICC'15), pp. 7228-7233, June 2015

[25] X. Xia, W. Liang, Y. Xiao, M. Zheng, and Z. Xiao, "Differencecomparison-based Approach for Malicious Meter Inspection in Neighborhood Area Smart Grids. In Proc. of the 50th International Conference on Communications (ICC'15), pp. 802-807, June 2015.

[26] W. Han and Y. Xiao, "NFD: A Practical Scheme to Detect Non-Technical Loss Fraud in Smart Grid," In Proc. 50th International Conference on Communications (ICC), pp. 605-09, Sydney, Australia, June 2014.

[27] D. Herrmann, "Lagrange-Interpolation," Numerische Mathematik - 40 BASIC-Programme, pp. 67-68, 1983.

[28] T. B Smith, "Electricity Theft: A Comparative Analysis," Energy Policy, Vol. 32, No. 18, pp. 2067-2076, Dec. 2004.

[29] R. F. Ghajar and J. Khalife, "Cost/benefit Analysis of an AMR System to Reduce Electricity Theft and Maximize Revenues for lectricit du Liban," Applied Energy, Vol. 76, No. 1-3, pp. 25-37, Sept. 2003.

[30] P. Kadurek, J. Blom, J. F. G. Cobben, and W. L. Kling, "Theft Detection and Smart Metering Practices and Expectations in the Netherlands," In Proc. Innovative Smart Grid Technologies Conference Europe, pp. 1-6, Oct. 2010.

[31] M. Golden and B. Min, "Theft and Loss of Electricity in an Indian State," available at: http://www.yale-university.com/leitner/resources/ papers/GM_PowerTheft_20120409.pdf, 2012.

[32] R. Passey, T. Spooner, I. MacGill, M. Watt, and K. Syngellakis, "The Potential Impacts of Grid-connected Distributed Generation and How to Address Them: A Review of Technical and Non-technical Factors," Energy Policy, Vol. 39, No. 10, pp. 6280-6290, Oct. 2011.

[33] D. Madan, M. Dhakla, and B. Dhull, "Effect of NTL on Power System Losses at Industrial Load," International Journal of Electronics Communication and Computer Engineering, Vol. 3, No. 4, pp. 773-778, Jul. 2012.

[34] F. M. Mwaura, "Adopting Electricity Prepayment Billing System to Reduce Non-technical Energy Losses in Uganda: Lesson from Rwanda," Utilities Policy, In Press, Jun. 2012.

[35] Northeast Group LLC, "Emerging Markets Smart Grid: Outlook 2015," 
available at: http://www.northeast-group.com/reports/BrochureEmerging\%20Markets\%20Smart\%20Grid-Outlook\%202015-

Northeast\%20Group.pdf, Dec. 2014.

[36] IEI Report, Utility-scale Smart Meter Deployments: Building Block of the Evolving Power Grid.

available at: http://www.edisonfoundation.net/iei/Documents/ IEI_SmartMeterUpdate_0914.pdf

[37] B. Gu, V. S. Sheng, K. Tay, W. Romano, and S. Li, "Incremental Support Vector Learning for Ordinal Regression," IEEE Transactions on Neural Networks and Learning Systems, vol. 26, no. 7, pp. 1403-1416, 2015.

[38] J. Nagi, K.S. Yap, F. Nagi, S.K. Tiong, S. P. Koh, and S. K. Ahmed, "NTL Detection of Electricity Theft and Abnormalities for Large Power Consumers in TNB Malaysia," In Proc. 2010 IEEE Student Conference on Research and Development (SCOReD), pp. 202-206, Dec. 2010.

[39] A.H. Nizar, "Customer Information System Data Pre-Processing with Feature Selection Techniques for Non-Technical Losses Prediction in an Electricity Market," In Proc. International Conference on Power System Technology (PowerCon), pp. 1-7, Chongqing, Oct. 2006.

[40] C. Ramos, A. Souza, G. Chiachia, A. Falco, and J. Papa, "A novel algorithm for feature selection using Harmony Search and its application for non-technical losses detection," Computers \& Electrical Engineering, Vol. 37, No. 6, pp. 886-894, Nov. 2011.

[41] C. Ramos, A. Souza, G. Chiachia, A. Falc, and J. Papa, "What is the importance of selecting features for non-technical losses identification?" In Proc. IEEE international symposium on circuits and systems, pp. 10451048, Rio de Janeiro, Brazil, 2011.

[42] F. Biscarri, I. Monedero, C. Leon, and J. I. Guerrero, "A Mining Framework to Detect Non-technical Losses in Power Utilities," In Proc. 11th International Conference on Enterprise Information System (ICEIS2009), 2009.

[43] B. C. Costa, B. L. A. Alberto, A. M. Portela, W. Maduro, and E. O. Eler "Fraud Detection in Electric Power Distribution Networks Using an Annbased Knowledge-discover Process, International Journal of Artificial Intelligence \& Applications (IJAIA), Vol. 4, No. 6, pp. 11-23, Nov. 2013.

[44] E. W. S. dos Angelos, O. R. Saavedra, O. A. C. Cortes, and A. N. de Souza, "Detection and Identification of Abnormalities in Customer Consumptions in Power Distribution Systems," IEEE Transactions on Power Delivery, Vol. 26, No. 4, pp. 2436-2442, Oct. 2011.

[45] Y. Lei, W. Jue, L. Feng, and P. Lingxi, "Research of privacy-preserving data aggregation algorithm for wireless sensor network," International Journal of Sensor Networks, Vol. 16, No. 1, 2014, pp. 41-47.

[46] F. Li, B. Luo, and P. Liu, "Secure and privacy-preserving information aggregation for smart grids," International Journal of Security and Networks, Vol. 6, No.1, pp. 28-39, 2011.

[47] G. Kalogridis, S.Z. Denic, T. Lewis, and R. Cepeda, "Privacy protection system and metrics for hiding electrical events," International Journal of Security and Networks, Vol. 6, No. 1, pp. 14-27, 2011.

[48] J. Liu, Y. Xiao, S. Li, W. Liang, and C. L. P. Chen, "Cyber Security and Privacy Issues in Smart Grids," IEEE Communications Surveys \& Tutorials, Vol. 14, No. 4, pp. 981-997, Fourth Quarter 2012.

[49] W. Han and Y. Xiao, "Privacy preserving for v2g networks in smart grid: A survey," Computer Communications, 2016. accepted. DOI: 10.1016j.comcom.2016.06.006

[50] J. Nagi, K. Yap, S. Tiong, S. Ahmed, and F. Nagi, "Improving SVMBased Nontechnical Loss Detection in Power Utility Using the Fuzzy Inference System," IEEE Transactions on Power Delivery, Vol. 26, No. 2, PP. 1284-1285, Apr. 2011

[51] X. Wen, L. Shao, Y. Xue, and W. Fang, "A rapid learning algorithm for vehicle classification," Information Sciences, vol. 295, no. 1, pp. 395-406, 2015.

[52] Depuru S.S.S.R., L. Wang, and V. Devabhaktuni, "Enhanced Encoding Technique for Identifying Abnormal Energy Usage Pattern," In Proc. North American Power Symposium (NAPS), pp. 1-6, Champaign, USA, Sept. 2012.

[53] A. H. Nizar, Z. Y. Dong, and Y. Wang, "Power Utility Nontechnical Loss Analysis With Extreme Learning Machine Method," IEEE Transaction On Power Systems, Vol. 23, No. 3, Aug. 2008.

[54] G.-B. Huang, Q.-Y. Zhu, and C.-K. Siew, "Extreme Learning Machine: A New Learning Scheme of Feedforward Neural Networks, In Proc. 2004 IEEE International Joint Conference of Neural Networks, 2004.

[55] J. I. Guerrero, C. Len, F. Biscarri, I. Monedero, J. Biscarri, and R. Milln, "Increasing the Efficiency in Non-Technical Losses Detection in Utility Companies," In Proc. 15th IEEE Mediterranean Electrotechnical Conference (MELECON), pp. 136-141, Valletta, Malta, Apr. 2010.

[56] B. Gu and V. S. Sheng, "A Robust Regularization Path Algorithm for ?-Support Vector Classification," IEEE Transactions on Neural Networks and Learning Systems, DOI : 10.1109/TNNLS.2016.2527796, 2016
[57] Y. Zheng, B. Jeon, D. Xu, Q.M. J. Wu, and H. Zhang, "Image segmentation by generalized hierarchical fuzzy C-means algorithm," Journal of Intelligent and Fuzzy Systems, vol .28, no. 2, pp. 961-973, 2015.

[58] B. Gu, V. S. Sheng, Z. Wang, D. Ho, S. Osman, and S. Li, "Incremental learning for ?-Support Vector Regression," Neural Networks, vol. 67, pp. 140-150, 2015.

[59] C. Ramos, A. Souza, J. Papa, and A. Falco, "Fast Non-Technical Losses Identification Through Optimum-Path Forest," In Proc. 15th International Conference on Intelligent System Applications to Power Systems(ISAP2009), pp. 1, Curitiba, Brazil, Nov. 2009.

[60] W. A. Doorduin, H. du T. Mouton, R. Herman, and H. J. Beukes, "Feasibility Study of Electricity Theft Detection Using Mobile Remote Check Meters," In Proc. 7th AFRICON Conference in Africa, Vol. 1, pp. 373-376, Sept. 2004.

[61] C. J. Bandim, J. E. R. Alves Jr, and A. V. Pinto Jr, "Identification of Energy Theft and Tampered Meters Using a Central Observer Meter: A Mathematical Approach," In Proc. IEEE/PES Transmission and Distribution Conference and Exposition, Vol. 1, pp. 163-168, Sept. 2003.

[62] I. Monedero, F. Biscarri, C. Len, J. Guerrero, and J. Biscarri, "Using Regression Analysis to Identify Patterns of Non-technical Losses on Power Utilities," Knowledge-based and Intelligent Information and Engineering Systems, Vol. 6276, pp. 410-419, 2010.

[63] Y. Lo, S. Huang, and C. Lu, "Non-technical Loss Detection Using Smart Distribution Network Measurement Data," In Proc. IEEE Innovative Smart Grid Technologies - Asia (ISGT Asia), Tianjin, China, May, 2012

[64] M. C. Evaldt, J. Santos, R. Figueiredo, L. Silva, and M. Stracke, "Payback Analysis in Identification and Monitoring of Commercial Losses in Distribution Networks," In Proc. 9th International Conference on the European Energy Market (EEM), pp. 1-6, Florence, Italy, May 2012.

[65] J. Gao, Y. Xiao, J. Liu, W. Liang, and C. L. P. Chen, "A Survey of Communication/Networking in Smart Grids," Future Generation Computer Systems, Vol. 28, No. 2, pp. 391-404, Feb. 2012.

[66] J. Lin, B. Zhu, P. Zeng, W. Liang, H. Yu, and Y. Xiao, "Monitoring Power Transmission Lines Using a Wireless Sensor Network," Wireless Communications and Mobile Computing (WCMC) Journal, John Wiley \& Sons, Vol. 15, No. 14, Jan. 2014

[67] J. Zhang and C. A. Gunter, "Application-aware secure multicast for power grid communications," International Journal of Security and Networks, Vol. 6, No. 1, pp. 40-52, 2011.

[68] D. Kundur, X. Feng, S. Mashayekh, S. Liu, T. Zourntos, and K.L. ButlerPurry, "Towards Modelling the Impact of Cyber Attacks on a Smart Grid," International Journal of Security and Networks, Vol. 6, No. 1, pp. 2-13, 2011.

[69] O. Osanaiyea, K. Choob, and M. Dlodlo, "Distributed denial of service (DDoS) resilience in cloud: Review and conceptual cloud DDoS mitigation framework," Journal of Network and Computer Applications, Vol. 67, pp. 147-165, May 2016.

[70] V. Prokhorenko, K. Choo, and H. Ashman, "Web application protection techniques: A taxonomy," Journal of Network and Computer Applications, Vol. 60, pp. 95-112, Jan. 2016.

[71] V. Prokhorenko, K. Choo, and H. Ashman, "Context-oriented web application protection model," Applied Mathematics and Computation, Vol. 285, pp. 59-78, July 2016.

[72] K. Choo, "The cyber threat landscape: Challenges and future research directions," Computers \& Security, Vol. 30, No. 8, pp. 719-731, Nov. 2011.

[73] Q. Do, B. Martini, and K. Choo, "Exfiltrating data from Android devices," Computers \& Security, Vol. 48, pp. 74-91, Feb. 2015.

[74] E. McCary and Y. Xiao, "Smart Grid Attacks and Countermeasures," EAI Endorsed Transactions on Industrial Networks and Intelligent Systems, Vol. 2, No. 2, pp. e4, Feb. 25, 2015, doi: 10.4108/inis.2.2.e4

[75] P. Jokar, N. Arianpoo, and V. C. M. Leung, "A Survey on Security Issues in Smart Grids," Security and Communication Networks, Vol. 9, No. 3, pp. 262-273, June 2012.

[76] N. Rahmana and K. Choo, "A survey of information security incident handling in the cloud," Computers \& Security, Vol. 49, pp. 45-69, Mar. 2015.

[77] Y. Ren, J. Shen, J. Wang, J. Han, and S. Lee, "Mutual Verifiable Provable Data Auditing in Public Cloud Storage," Journal of Internet Technology, vol. 16, no. 2, pp. 317-323, 2015.

[78] J. Liu, Y. Xiao, and J. Gao, "Achieving Accountability in Smart Grids," IEEE Systems Journal, Vol. 8, No. 2, pp. 493-508, June 2013.

[79] E. McCary and Y. Xiao, "Home Area Network Accountability with Varying Consumption Devices in Smart Grid," (Wiley Journal of) Security and Communication Networks, Vol. 9, No. 10, pp. 977995, 10 July 2016. 
[80] W. Han and Y. Xiao, "IP2DM for V2G networks in smart grid," In Proc. of the 2015 International Conference on Communications (ICC'15), pp. 782-787, June 2015.

[81] Average household electricity use around the world. available at: http://shrinkthatfootprint.com/average-household-electricity-consumption (2010).

[82] M. Spivak, Calculus (3rd ed.), Houston, TX: Publish or Perish, p. 383 , 1994.

[83] M. Comenetz, Calculus: The Elements. World Scientific. p. 159, 2002.

[84] X. Bai, "Study of Taylor Formular Approximation Precision," College Mathematics, Vol.2, No. 4, pp. 108-110, Aug. 2004.

[85] Runge's phenomenon - Tallinn University. (n.d.).

Available at: http://www.tlu.ee/ tonu/Arvmeet/Runge's\%20phenomenon.pdf

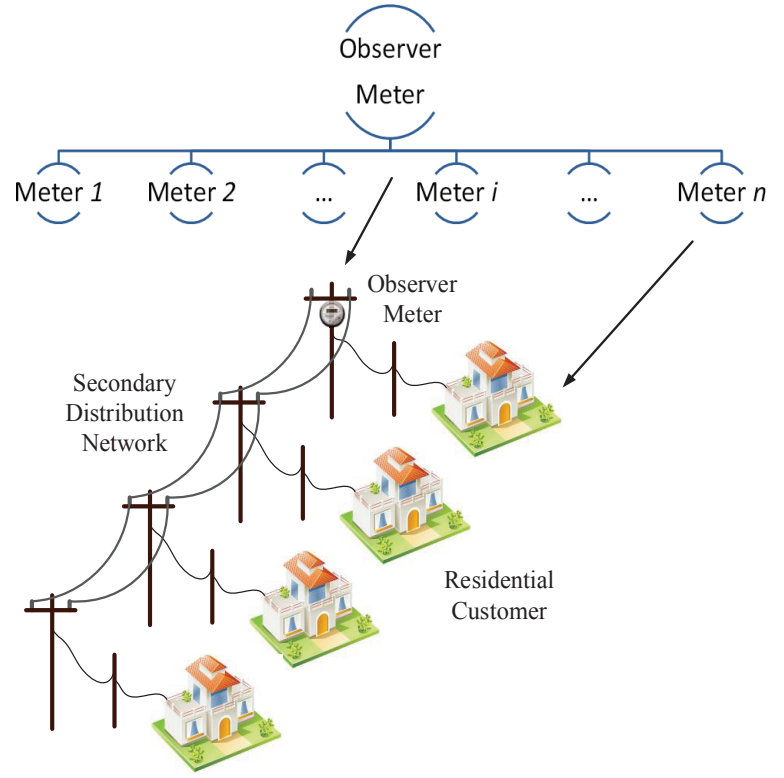

Fig. 1. The conceptual framework of NTL frauds. A simplified model of the metering system: an observer meter records the total electricity supplied to $n$ meters under its observation.

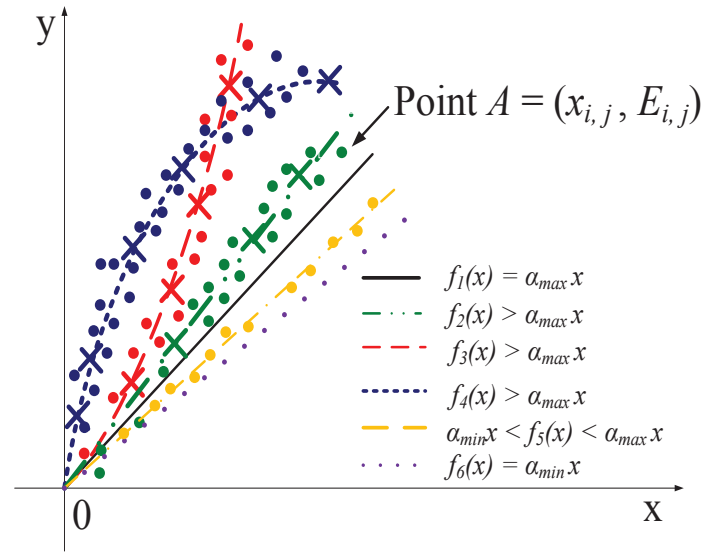

Fig. 2. Points on the Coordinate, with the corresponding value pairs (the measured electricity, the consumed electricity). Curves of the normal meters and the possibly tampered ones. The black line is of the normal meter. Other curves above this line are different potential curves of the tampered meters according to Corollary 1.

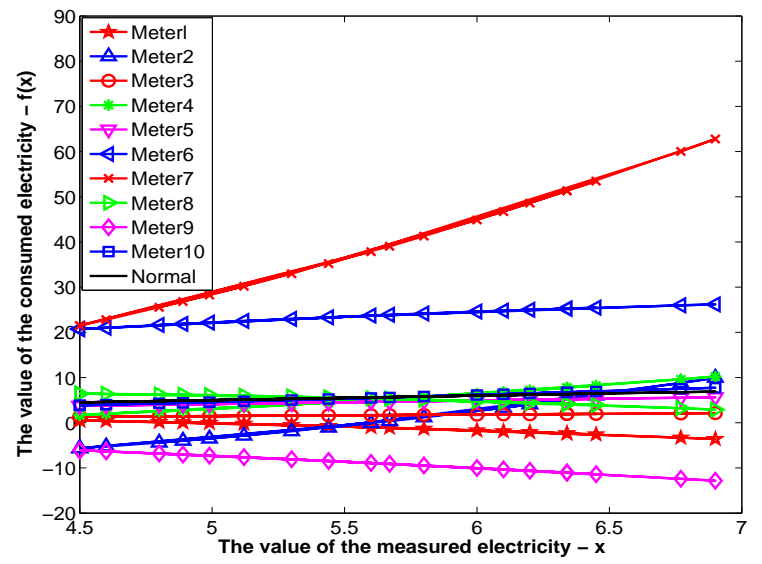

Fig. 4. The curves of the polynomials in the experiment of lowering order. A false alarm occurs when using an order- 2 polynomial instead of an order- 3 polynomial.

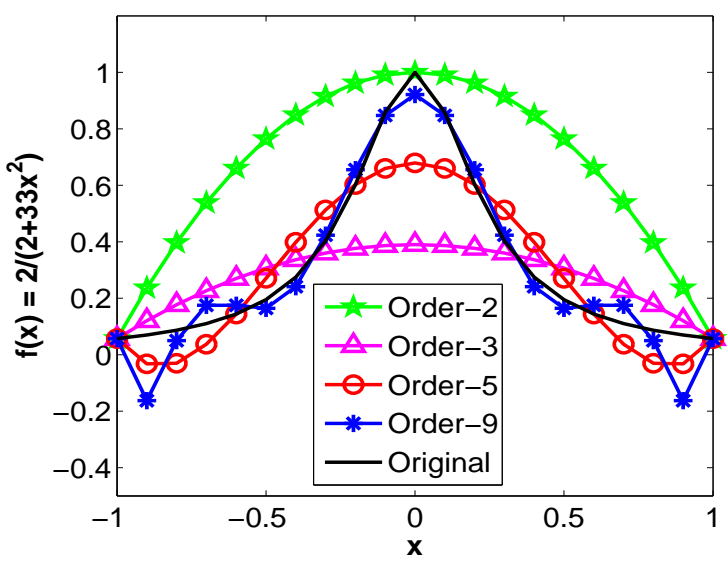

Fig. 5. The curves of approximation polynomials with different orders of the same function $f_{i}(x)=\frac{2}{2+33 x^{2}}$. A counter example to illustrate a higher order does not always improve accuracy. 


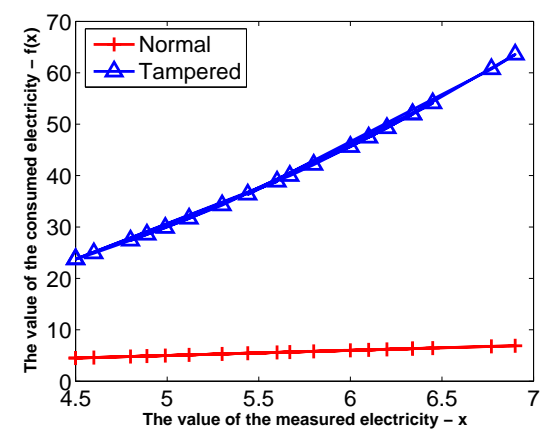

(a)

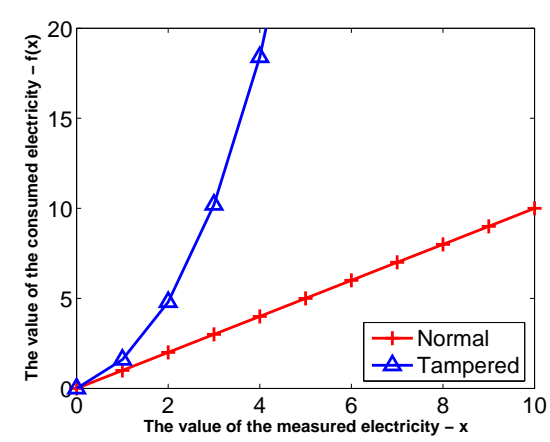

(b)

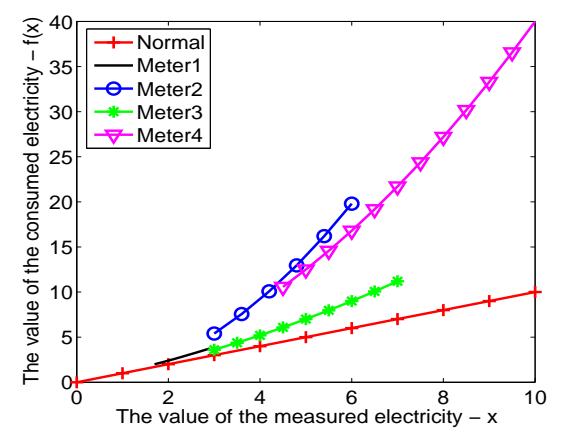

(c)

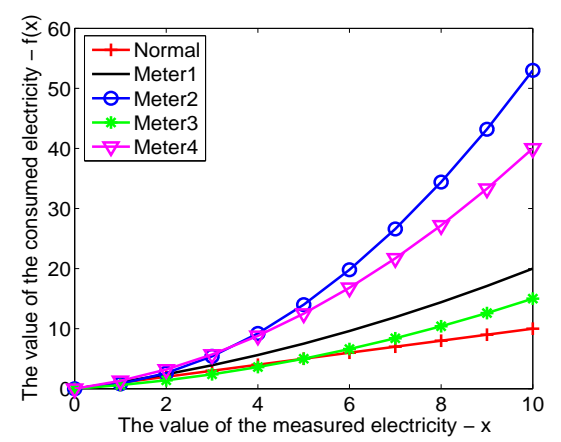

(d)

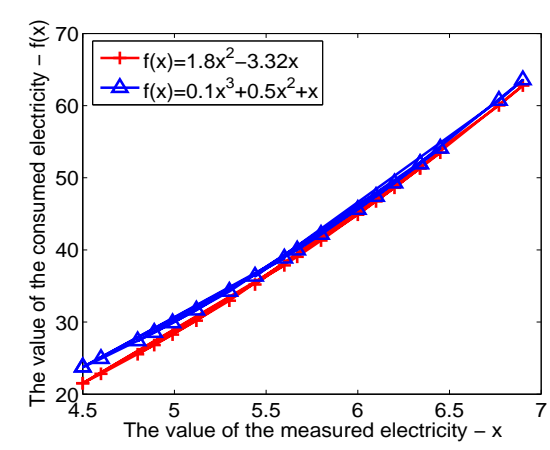

(e)

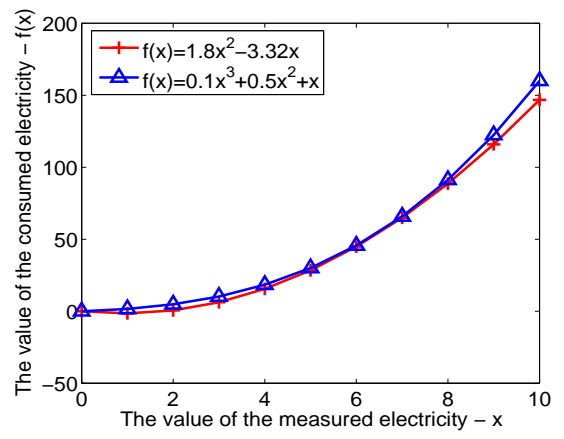

(f)

Fig. 3. Experimental results of all the experiments in this paper. The range of $x$ shown in a), c) and e) is obtained from the related dataset. The range of $x$ in b), d) and f) is extended to [0,10]. (a)(b) The curves of all the polynomials in Experiment 2. (c)(d) The curves of all the polynomials in Experiment 3. (e)(f) Error comparison between the order 2 and order 3 polynomials of the same meter - Meter 7, obtained from the same dataset in the experiment. 